\title{
Erratum to: Tomotherapy PET-guided dose escalation - A dosimetric feasibility study for patients with malignant pleural mesothelioma
}

\author{
Angelo Maggio - Claudia Cutaia - Amalia Di Dia - Sara Bresciani · \\ Anna Miranti · Matteo Poli · Elena Delmastro · Elisabetta Garibaldi · \\ Pietro Gabriele $\cdot$ Michele Stasi
}

Published online: 6 November 2015

(C) Springer-Verlag Berlin Heidelberg 2015

Erratum to: Strahlenther Onkol (2015) 191

doi:10.1007/s00066-015-0901-8

Unfortunately, erroneous author affiliations were published in the article "Tomotherapy PET-guided dose escalation A dosimetric feasibility study for patients with malignant pleural mesothelioma"

The correct list of author affiliations reads as follows:

Angelo Maggio ${ }^{1}$, Claudia Cutaia ${ }^{1}$, Amalia Di Dia ${ }^{1}$,

Sara Bresciani ${ }^{1}$, Anna Miranti ${ }^{1}$, Matteo Poli ${ }^{1}$,

Elena Delmastro ${ }^{2}$, Elisabetta Garibaldi ${ }^{2}$, Pietro Gabriele ${ }^{2}$

and Michele Stasi ${ }^{1}$

${ }^{1}$ Medical Physics Department, Candiolo Cancer InstituteFPO, IRCCS, Turin, Italy

${ }^{2}$ Radiotherapy Department, Candiolo Cancer Institute FPO, IRCCS, Turin, Italy

We apologize for any inconveniences caused.

The online version of the original article can be found at doi:10.1007/s00066-015-0901-8

\footnotetext{
A. Maggio $(\bowtie) \cdot$ C. Cutaia $\cdot$ A. D. Dia $\cdot$ S. Bresciani ·

A. Miranti $\cdot$ M. Poli $\cdot$ M. Stasi

Medical Physics Department, Candiolo Cancer Institute, FPO,

IRCCS,

Turin, Italy

e-mail: maggio.angelo@gmail.com

E. Delmastro $\cdot$ E. Garibaldi $\cdot$ P. Gabriele

Radiotherapy Department, Candiolo Cancer Institute - FPO,

IRCCS,

Turin, Italy
} 